\title{
Coupling of Local and Global Quantities by A Subproblem Finite Element Method - Application to Thin Region Models
}

\author{
Dang Quoc Vuong ${ }^{*}, 1$ and Nguyen Duc Quang ${ }^{2}$ \\ ${ }^{1}$ School of Electrical Engineering, Ha Noi University of Science and Technology, Viet Nam \\ ${ }^{2}$ Faculty of Electrical Engineering, Electric Power University, Hanoi, Viet Nam
}

\begin{tabular}{l} 
A R T I C L E I N F O \\
\hline Article history: \\
Received: 29 October, 2018 \\
Accepted: 10 January, 2019 \\
Online: 07 March, 2019
\end{tabular}

Keywords:

Finite element method (FEM)

Magnetostatics

Magnetodynamics

Subproblem method (SPM)

Coupled problems thin region

Magnetic fields

\begin{abstract}
A B S T R A C T
A method for coupling of local and global fields related to currents, voltages and magnetic fields in magnetodynamic problems is developed in the frame of the finite subproblem finite element method. The method allows to correct the errors arising from thin conducting regions, that replace volume thin regions by surfaces but neglect border effects in the vicinity of geometrical discontinuities, edges and corners, increasing with the thickness, which limits their range of validity. This leads to errors when solving the thin shell finite element magnetic models in electrical machines and devices. It also permits to perform a natural coupling between local and global quantities weak formulations. A subproblem finite element method is developed to split a complete problem/model composed of local and global fields (some of these being thin regions) into a series of subproblems with the change of materials. Each subproblem is performed on its own separate domain and mesh.
\end{abstract}

\section{Introduction}

Many papers have been published about thin region finite element (FE) models [1]-[7]. Besides theoretical studies on the shielding effect and related to interface conditions (ICs), several finite element (FE) subproblem method (SPM) formulations for the thin shell (TS) models have been developed [1]-[7]. This means that instead of meshing the volume thin regions, the TS models can be considered as surfaces with ICs, that neglect errors on the computation of local electromagnetic quantities in the vicinity of geometrical discontinuities, edges and corners, increasing with the thickness [1] - [4].

In this paper, the FE SPM is herein extended for coupling of local fields (magnetic flux density, magnetic field and eddy current) and global quantities (currents, voltages Joule losses) associated with any conducting part of an electric system to correct the inherent errors of the local and global fields near eges and corners comming from the TS models. It is particularly the case with inductors driven by external sources. For such components, either a global current $I_{i}$ or a global voltage $V_{i}$ can be fixed (Figure 1 ), in a more general way, both of them must be taken into account when a coupling with circuit equation is performed.

\footnotetext{
*Dang Quoc Vuong, Hanoi Universtiy of Science and Technology,

Email: Vuong.dangquoc@hust.edu.vn
}

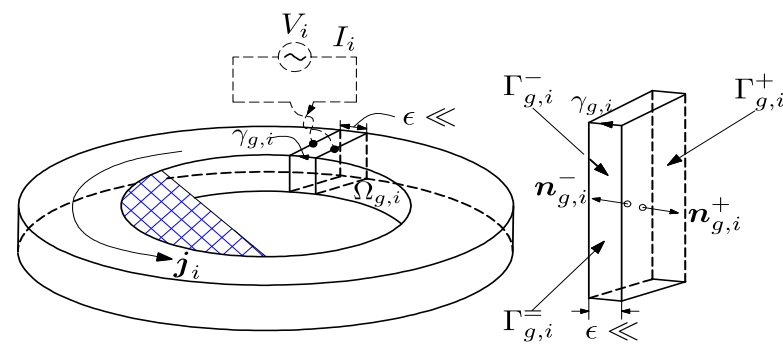

Figure 1. Generator $\Omega_{\mathrm{g}, \mathrm{i}}$ with associated global current $I_{i}$ and voltage $V_{i}$.

The FE SPM allows to couple any changes from this problem to others via surface sources (SSs) and volume sources (VSs) and applied via a projection method [1]-[4]. The development of the method is proposed for the magnetic vector potential FE magneto dynamic formulation, paying special attention to the proper discretization of the constraints involved in each subproblem (SP) and to the resulting weak FE formulations. The method is validated on a practical test problem [8].

\section{Coupled Magnetic Subproblem}

\subsection{Sequence of Subproblems}

At the hearth of the SPM, a full problem is proposed to be divided into sequences of SPs: A problem involving global current driven stranded or massive inductors alone is first performed on a simplified mesh without any thin regions. The obtained solution 
considered as SSs for added TS problems via ICs [1] - [7]. The TS solution is then corrected by a correction problem via SSs and VSs, that overcomes the TS assumptions as presented in Section 1.

\subsection{Canonical magnetodynamic problem}

A canonical 3-D magnetodyanmic problem $i$, to be solved at step $i$ of the SPM, is defined in a domain $\Omega_{i}$, with boundary $\partial \Omega_{i}=\Gamma_{i}=\Gamma_{h, i} \cup \Gamma_{b, i}$. The eddy current defined in the conducting part and the non-conducting of $\Omega_{i}$ are respectively denoted $\Omega_{c, i}$ and $\Omega_{c, i}^{C}$, with $\Omega_{i}=\Omega_{c, i} \cup \Omega_{c, i}^{C}$. Stranded inductors belong to $\Omega_{c, i}^{C}$, whereas massive inductors belong to $\Omega_{c, i}$. The equations and material relations of SPs $i$ are [9] - [11]:

$$
\begin{gathered}
\operatorname{curl} \boldsymbol{h}_{i}=\boldsymbol{j}_{i}, \quad \operatorname{div} \boldsymbol{b}_{i}=0, \operatorname{curl} \boldsymbol{e}_{i}=-\boldsymbol{\partial}_{t} \boldsymbol{b}_{i} \quad(1 \mathrm{a}-\mathrm{b}-\mathrm{c}) \\
\boldsymbol{h}_{i}=\mu_{i}^{-1} \boldsymbol{b}_{i}+\boldsymbol{h}_{s, i}, \quad \boldsymbol{j}_{i}=\sigma_{i} \boldsymbol{e}_{i}+\boldsymbol{j}_{s, i}, \quad \text { (2a-b) }
\end{gathered}
$$

where $\boldsymbol{h}_{i}$ is the magnetic field, $\boldsymbol{b}_{i}$ is the magnetic flux density, $\boldsymbol{e}_{i}$ is the electric field, $\boldsymbol{j}_{i}$ is the electric current density, $\mu_{i}$ is the magnetic permeability and $\sigma_{i}$ is the electric conductivity. Note that (1c) is only defined in $\Omega_{c, i}$, whereas it is reduced to the form (1b) in $\Omega_{c, i}^{C}$. Boundary conditions (BCs) are defined on complementary parts $\Gamma_{h, i}$ and $\Gamma_{b, i}$, i.e.

$$
\boldsymbol{n} \times \boldsymbol{h}_{i}=\boldsymbol{j}_{f, i}, \quad \boldsymbol{n} \times\left.\boldsymbol{b}_{i}\right|_{\Gamma_{b, i}}=\boldsymbol{f}_{f, i},
$$

where $\boldsymbol{n}$ is the unit normal exterior to $\Omega_{i}$. The surface fields $\boldsymbol{j}_{f, i}$ and $\boldsymbol{f}_{f, i}$ in (3a) and (3b) are SSs which comes from previous problems [2] - [6]. For the classical homogeneous BCs, they normally define as zero. However, they define as possible SSs in the thin region between $\gamma_{i}^{+}$and $\gamma_{i}^{-}$[1] - [7]. This is the case when some field traces in a $\mathrm{SP}_{u}$ are forced to be discontinuous, whereas their continuity must be recovered via a $\mathrm{SP}_{p}$. The $\mathrm{SSs}_{\text {in }} \mathrm{SP}_{p}$ are thus to be fixed as the opposite of the trace solution of $\mathrm{SP}_{u}$.

In addition, global conditions on currents or voltages in inductors are considered. A typical inductor is shown in Figure 1 where a source of electromotive force $\Omega_{g, i}$ is defined between two electrodes very close to each other of voltage $V_{i}$ and the current $I_{i}$ following through surface $\Gamma_{g, i}$, i.e.

$$
\oint_{\Gamma_{i}^{-}}^{\Gamma_{i}^{+}} \boldsymbol{e} \cdot d l=V_{i} \text { and } \oint_{\Gamma_{i}^{-}}^{\Gamma_{i}^{+}} n \cdot \boldsymbol{j} d s=I_{i},
$$

where $\Gamma_{i}$ is a path in $\Omega_{g, i}$ connecting its two electrodes. Surface $\Gamma_{g, i}$ is defined as a part of the boundary $\Gamma_{e, i}$ of the studied domain in presences symmetry conditions.

The fields $\boldsymbol{h}_{s, i}$ and $\boldsymbol{j}_{s, i}$ (fixes the global current in inductors) and in (2a) and (2b) are VSs which can be used for expressing changes of materials in each SP [1]-[4]. Indeed, for changes of permeability and conductivity in a region, from $\mathrm{SP}_{u}(i=\mathrm{u})$ to $\mathrm{SP}_{p}(i=\mathrm{p})$ are defined via VSs $\boldsymbol{h}_{s, i}$ and $\boldsymbol{j}_{s, i}$, i.e.

$$
\boldsymbol{h}_{s, p}=\left(\mu_{p}^{-1}-\mu_{u}^{-1}\right) \boldsymbol{b}_{u}, \quad \boldsymbol{j}_{s, p}=\left(\sigma_{p}-\sigma_{u}\right) \boldsymbol{e}_{u}
$$

Each SP is constrained through the so defined SSs and VSs from the parts of the solutions of other SPs.

\section{Finite Element Weak Formulation}

\subsection{Magnetic Vector Potential Formulation}

We can define a vector potential $\boldsymbol{a}_{i}$ so that $\boldsymbol{b}_{i}=\operatorname{curl} \boldsymbol{a}_{i}$ and $\boldsymbol{e}_{i}=\sigma_{i} \partial_{i} \boldsymbol{a}_{i}-\sigma_{i} \operatorname{grad} v_{i}$. A weak formulation of $\mathrm{SP}_{i}(i=u, p$ or $k)$ of the Ampère equation (1a) can be written as [1]-[4].

www.astesj.com

$$
\begin{aligned}
\left(\mu_{i}^{-1} \operatorname{curl} \boldsymbol{a}_{i}, \operatorname{curl} \boldsymbol{a}_{i}^{\prime}\right)_{\Omega_{i}}+\left(\boldsymbol{h}_{s, i}, \operatorname{curl} \boldsymbol{a}_{i}^{\prime}\right)_{\Omega_{i}}+\left(\boldsymbol{j}_{s, i}, \boldsymbol{a}_{i}^{\prime}\right)_{\Omega_{i}} \\
+\left(\sigma_{i} \partial_{i} \boldsymbol{a}_{i}, \boldsymbol{a}_{i}^{\prime}\right)_{\Omega_{c, i}}+\left(\sigma_{i} \operatorname{grad} v_{i}, \boldsymbol{a}_{i}^{\prime}\right)_{\Omega_{c, i}} \\
+<\boldsymbol{n} \times \boldsymbol{h}_{i}, \boldsymbol{a}_{i}^{\prime}>_{\Gamma_{h, i}-\Gamma_{t, i}} \\
+<\left[\boldsymbol{n} \times \boldsymbol{h}_{i}\right]_{\Gamma_{t, i}}, \boldsymbol{a}_{i}^{\prime}>_{\Gamma_{t, i}} \\
\quad=\left(\boldsymbol{j}_{i}, \boldsymbol{a}_{i}^{\prime}\right)_{\Omega_{s, i}}, \forall \boldsymbol{a}_{i}^{\prime} \in F_{i}^{1}\left(\Omega_{i}\right)
\end{aligned}
$$

where $F_{i}^{1}(\Omega)$ is a curl-conform function space defined in $\Omega_{c, i}$, gauged in $\Omega_{c, i}^{C}$, and containing the basis functions for $\boldsymbol{a}_{i}$ as well as for the test function $\boldsymbol{a}_{i}^{\prime}$ (at the discrete level, this space is defined by edge FEs; the gauge is based on the tree-co-tree technique); $(\cdot, \cdot)_{\Omega}$ and $\langle\cdot, \cdot\rangle_{\Gamma}$ respectively denote a volume integral in $\Omega$ and a surface integral on $\Gamma$ of the product of their vector field arguments. The surface integral term on $\Gamma_{h, i}$ is considered as natural BCs of type (3a), usually zero. The electrical scalar potential $v_{i}$ is only defined in the conducting regions $\Omega_{c, i}$. The weak formulation (6) implies, by taking $\boldsymbol{a}_{i}^{\prime}=\operatorname{grad} v_{i}^{\prime}$ as a test function, that

$$
\begin{gathered}
\left(\sigma_{i} \partial_{t} \boldsymbol{a}_{i}, \operatorname{grad} v_{i}^{\prime}\right)_{\Omega_{c, i}}+\left(\sigma_{i} \operatorname{grad} v_{i}, \operatorname{grad} v_{i}^{\prime}\right)_{\Omega_{c, i}} \\
+\left(\boldsymbol{h}_{s, i}, \operatorname{curl} \boldsymbol{a}_{i}^{\prime}\right)_{\Omega_{i}}+\left(\boldsymbol{j}_{s, i}, \boldsymbol{a}_{i}^{\prime}\right)_{\Omega_{i}} \\
+<\left[\boldsymbol{n} \times \boldsymbol{h}_{i}\right]_{\Gamma_{t, i}}, \boldsymbol{a}_{i}^{\prime}>_{\Gamma_{t, i}}=<\boldsymbol{n} \cdot \boldsymbol{j}, v_{i}^{\prime}>_{\Gamma_{g}}, \forall \boldsymbol{a}_{i}^{\prime} \in F_{i}^{1}\left(\Omega_{i}\right)
\end{gathered}
$$

where $\Gamma_{g}$ is the part of the boundary of $\Omega_{c, i}$ which is crossed by a current $\left(\Gamma_{g}\right.$ is the union of all the surfaces $\Gamma_{g, i}$ resulting from the abstraction of the generators $\Omega_{g, i}$ ) (Fig. 1).

\subsection{Current as weak global quantities and circuit relations}

For the weak formulation in (6), the appearing of total current in a conductor is only expressed in a weak sense, e.g. as a natural global constraint, because it arises from Ampère law which is its self-expressed in a weak form. By solving the equation (7), the current $I_{i}$ flowing in the part $\Gamma_{g, i}$ of an inductor can be obtained with $v_{i}^{\prime}$ equal to the source scalar potential $v_{s, i}$. Hence, with $v_{i}^{\prime}=$ $v_{s, i}$, the surface intergral term in (7) written for the inductor $\Omega_{m, i}$ gives

$$
<\boldsymbol{n} \cdot \boldsymbol{j}, v_{s, i}>_{\Gamma_{g, i}}=<\boldsymbol{n} \cdot \boldsymbol{j}, 1>_{\Gamma_{g, i}}=I_{i},
$$

and thus (7) becomes

$$
\begin{aligned}
&\left(\sigma_{i} \partial_{i} \boldsymbol{a}_{i}, \operatorname{grad} v_{s, i}\right)_{\Omega_{m, i}}+\left(\sigma_{i} \operatorname{grad} v_{i}, \operatorname{grad} v_{s, i}\right)_{\Omega_{m, i}} \\
&+\left(\boldsymbol{h}_{s, i}, \operatorname{curl} \boldsymbol{a}_{i}^{\prime}\right)_{\Omega_{i}}+\left(\boldsymbol{j}_{s, i}, \boldsymbol{a}_{i}^{\prime}\right)_{\Omega_{i}} \\
&+\quad<\left[\boldsymbol{n} \times \boldsymbol{h}_{i}\right]_{\Gamma_{t, i}} \boldsymbol{a}_{i}^{\prime}>_{\Gamma_{t, i}}=I_{i},
\end{aligned}
$$

or, with $v=V_{i} v_{s, i}$

$$
\begin{aligned}
&\left(\sigma_{i} \partial_{i} \boldsymbol{a}_{i}, \operatorname{grad} v_{s, i}\right)_{\Omega_{m, i}}+V_{i}\left(\sigma_{i} \operatorname{grad} v_{s, i}, \operatorname{grad} v_{s, i}\right)_{\Omega_{m, i}} \\
&+\left(\boldsymbol{h}_{s, i}, \operatorname{curl} \boldsymbol{a}_{i}^{\prime}\right)_{\Omega_{i}}+\left(\boldsymbol{j}_{s, i}, \boldsymbol{a}_{i}^{\prime}\right)_{\Omega_{i}} \\
&+\quad<\left[\boldsymbol{n} \times \boldsymbol{h}_{i}\right]_{\Gamma_{t, i}}, \boldsymbol{a}_{i}^{\prime}>_{\Gamma_{t, i}}=I_{i} .
\end{aligned}
$$

Equation (10) is the circuit relation associated with the inductor $\Omega_{m, i}$, i.e. a relation between its voltage $V_{i}$ and its current $I_{i}$.

\subsection{Inductor alone "SP $\boldsymbol{P}_{\boldsymbol{u}}$ " - The added TS model $" \boldsymbol{S} \boldsymbol{P}_{\boldsymbol{p}}$ " - Volume correction "S $\boldsymbol{P}_{\boldsymbol{k}}$ "}

The weak form of an $\mathrm{SP}_{u}$ with the inductor alone is first solved via the volume integrals in $(10)(i \equiv u)$ where $I_{u}$ is the fixed current density in on $\Omega_{m, i}$, i.e.

$\left(\sigma_{i} \partial_{i} \boldsymbol{a}_{i}, \operatorname{grad} v_{s, i}\right)_{\Omega_{m, i}}+V_{i}\left(\sigma_{i} \operatorname{grad} v_{s, i}, \operatorname{grad} v_{s, i}\right)_{\Omega_{m, i}}=I_{i}$. 
The added TS problem is defined via the second term in (11) $(i \equiv$ $p)$. The test function $\boldsymbol{a}_{p}^{\prime}$ is divided into continuous and discontinuous parts $\boldsymbol{a}_{c, p}^{\prime}$ and $\boldsymbol{a}_{d, p}^{\prime}$ (with $\boldsymbol{a}_{d, p}^{\prime}$ zero on $\Gamma_{p}^{-}$) [6]. One thus has

$$
\begin{aligned}
<\left[\boldsymbol{n} \times \boldsymbol{h}_{p}\right]_{\Gamma_{t, p}}, \boldsymbol{a}_{p}^{\prime} & >_{\Gamma_{t, p}}=<\left[\boldsymbol{n} \times \boldsymbol{h}_{p}\right]_{\Gamma_{t, p}}, \boldsymbol{a}_{c, p}^{\prime}>_{\Gamma_{t, p}}+ \\
& <\boldsymbol{n} \times\left.\boldsymbol{h}_{p}\right|_{\Gamma_{t, p}^{+}}, \boldsymbol{a}_{d, p}^{\prime}>_{\Gamma_{t, p}^{+}}
\end{aligned}
$$

The terms of the right-hand side of (12) are developed from the TS models [6], i.e.

$$
\begin{gathered}
<\left[\boldsymbol{n} \times \boldsymbol{h}_{p}\right]_{\Gamma_{t, p}}, \boldsymbol{a}_{c, p}^{\prime}>_{\Gamma_{t, p}}=<\sigma_{p} \beta_{p} \partial_{t}\left(2 \boldsymbol{a}_{c, p}+\boldsymbol{a}_{d, p}\right), \boldsymbol{a}_{c, p}^{\prime}>_{\Gamma_{t, p}} \\
<\boldsymbol{n} \times\left.\boldsymbol{h}_{p}\right|_{\Gamma_{t, p}^{+}}, \boldsymbol{a}_{d, p}^{\prime}>_{\Gamma_{t, p}^{+}}=-<\boldsymbol{n} \times\left.\boldsymbol{h}_{u}\right|_{\Gamma_{t, p}^{+}}, \boldsymbol{a}_{d, p}^{\prime}>_{\Gamma_{t, p}^{+}} \\
+\frac{1}{2}<\sigma_{p} \beta_{p} \partial_{t}\left(2 \boldsymbol{a}_{c, p}+\boldsymbol{a}_{d, p}\right)+1 / \sigma_{p} \beta_{p}, \boldsymbol{a}_{d, p}^{\prime}>_{\Gamma_{t, p}} .
\end{gathered}
$$

The surface integral term $-<\boldsymbol{n} \times\left.\boldsymbol{h}_{u}\right|_{\Gamma_{t, p}^{+},}, \boldsymbol{a}_{d, p}^{\prime}>_{\Gamma_{t, p}^{+}}$in (14) is considered as a SS appeared from the weak formulation of $\mathrm{SP}_{u}$ (6), i.e.

$$
\begin{aligned}
-<\boldsymbol{n} \times\left.\boldsymbol{h}_{u}\right|_{\Gamma_{t, p}^{+}}, \boldsymbol{a}_{d, p}^{\prime} & >_{\Gamma_{t, p}^{+}}=\left(\sigma_{u} \partial_{u} \boldsymbol{a}_{u}, \operatorname{grad} v_{s, u}\right)_{\Omega_{m, p^{+}}} \\
& +V_{u}\left(\sigma_{u} \operatorname{grad} v_{s, u} \operatorname{grad} v_{s, u}\right)_{\Omega_{m, p^{+}}} .
\end{aligned}
$$

At the discrete level, the first term on the right side of (15) is thus limited to a single layer of FEs on the side $\Omega_{m, p^{+}}$touching $\Gamma_{t, p}^{+}$, because it occurs only the associated trace $\boldsymbol{n} \times\left.\boldsymbol{a}_{d, p}^{\prime}\right|_{\Gamma_{t, p}^{+}}$. Moreover, the source $\boldsymbol{a}_{u}$ obtained from the mesh of $\mathrm{SP}_{u}$ is then projected on the mesh of $\mathrm{SP}_{p}$ via a projection method [1] - [7].

Once achieved, the errors on the $\mathrm{TS} \mathrm{SP}_{p}$ solution is then corrected by $\operatorname{SP}_{k} \quad(i \equiv k)$ via the volume integrals $\left(\boldsymbol{h}_{s, k}, \operatorname{curl} \boldsymbol{a}_{k}^{\prime}\right)_{\Omega_{k}}$ and $\left(\boldsymbol{j}_{s, k}, \boldsymbol{a}_{k}^{\prime}\right)_{\Omega_{k}}$ in (10). The VSs $\boldsymbol{h}_{s, k}$ and $\boldsymbol{j}_{s, k}$ are given in (4a-b). In parallel with the VSs in (10), ICs recover the TS discontinuities to remove the TS representation via SSs opposed to previous TS ICs.
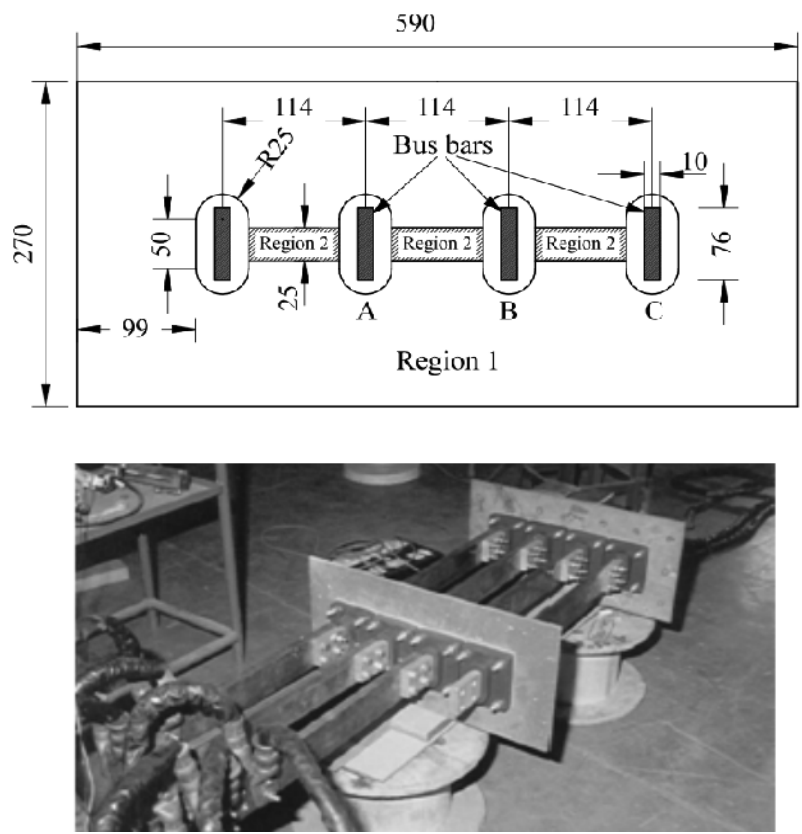

Figure 2. The geometry of the cover plate (top) and the experimental set-up (bottom) (all dimensions are in $\mathrm{mm}$ ).

\section{Application example}

The illustrate and validate the SPM with global quantities, an actual test problem is a cover plate of a transformer with ratings from 500kVA upto 2000kVA. The geometry of the cover plate is shown in Figure 2 (top) and the experimental set - up developed by the authors in [8] is presented in Figure 2 (bottom).

The three bus bars carry adjustable balanced three - phase currents up to $I_{a}=I_{\max } \sin (\omega t+0), I_{b}=I_{\max } \sin \left(\omega t-\frac{2 \pi}{3}\right)$ and $I_{c}=I_{\max } \sin (\omega t+2 \pi / 3)$. The distance between plates is $114 \mathrm{~mm}$ and the plate dimensions are 270x590x6mm (Figure 2, top). The cover plate is made of two different regions and properties (magnetic and non-magnetic). The conductivities for the regions 1 and 2 are taken as $\sigma_{1}=4.07 \mathrm{MS} / \mathrm{m}$ and $\sigma_{2}=1.15 \mathrm{MS} / \mathrm{m}$ respectively and the relative permeabilties for the regions 1 and 2 are taken as $\mu_{r, 1}=300$ and $\mu_{r, 1}=1$, respectively.

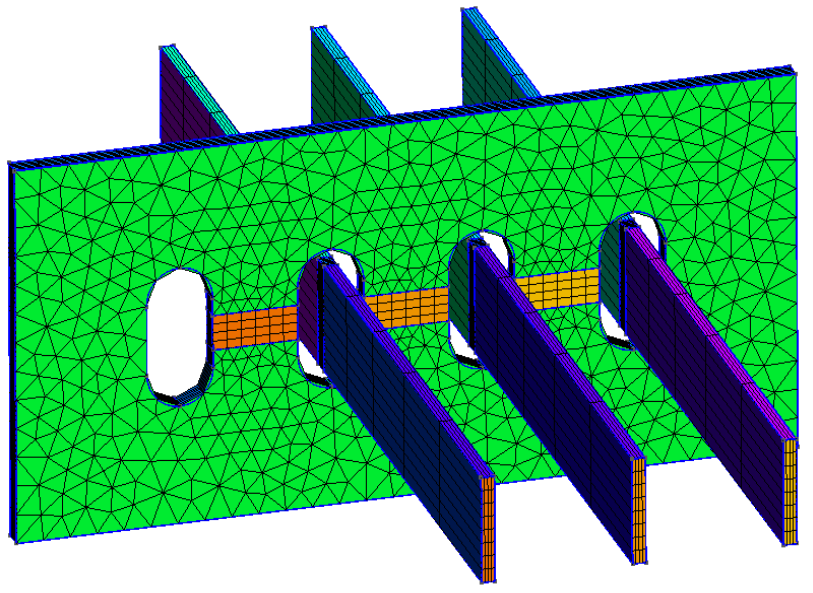

The problem herein is considered three SPs strategy. The field $\boldsymbol{b}_{u}$ computed in a simplified mesh in $\mathrm{SP}_{u}$ (with the bus bars considered as massive inductors) is shown in Figure 4 (top). A TS model SP $p$ (presenting the distribution of eddy current density $\boldsymbol{j}_{p}$ on the surface) is then added (Figure 4, middle). Finally, a $\mathrm{SP}_{k}$ replaces the TS model with volume correction covered by the actual plate and its neighborhood with an adequate refined mesh (Figure 4 , bottom). By integrating the value of $\boldsymbol{j}_{k}$ (Figure 4, bottom) along the thickness of the cover plate and comparing the result to the TS solution $\boldsymbol{j}_{p}$, it is obtained. Thus, the TS inaccuracy on $\boldsymbol{j}_{p}$ can locally reach $47 \%$ (Fig. 4 , middle $)(\mathrm{d}=6 \mathrm{~mm}, \mathrm{f}=50 \mathrm{~Hz}$, skindepth $\delta=2 \mathrm{~mm}$. The error on $\mathrm{TS} \mathrm{SP}_{p}$ solution through the cover plate hole (Figure 5), and along the cover plate border and near the plate ends (Figure 6) can reach 37,5\% and 50\%, respectively. The Joule losses and the global currents flowing in the bus bars calculated for the mounting plate (with non-magnetic (region 2) inserted) by the SPM and the experimental method proposed by authors in [8], are given in Table 1. It can be shown that there is a very good agreement. 

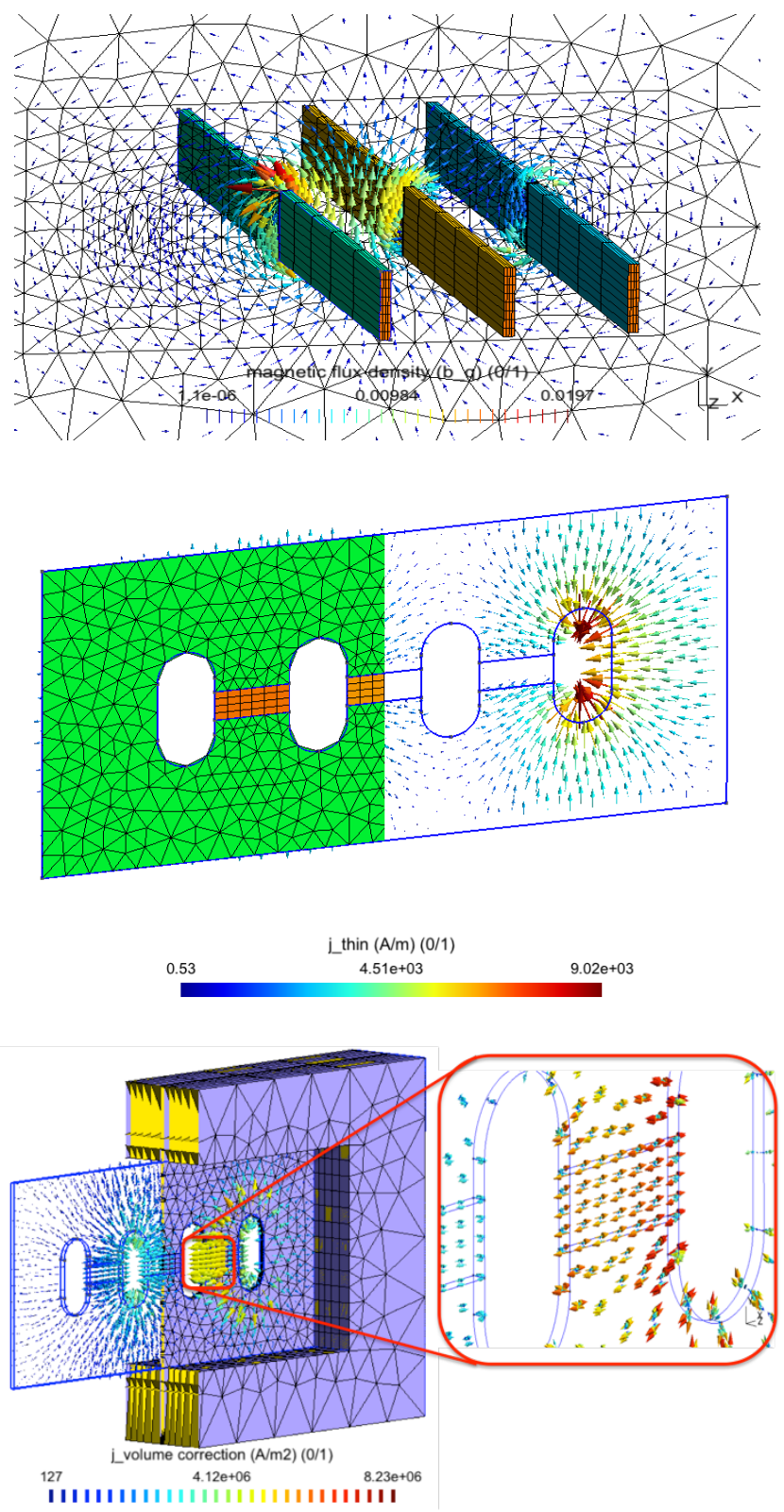

Figure 4. Magnetic flux density $b_{u}$ (in a cut plane) generated by massive inductors (top), TS eddy current $j_{p}$ (middle) and its volume correction $j_{k}$ (bottom) (thickness $\mathrm{d}=6 \mathrm{~mm}$, frequency $\mathrm{f}=50 \mathrm{~Hz}$ ).

This test problem has helped to standardize the type and material of the cover plate for various current in transformers rated between 50kVA upto 2000kVA.

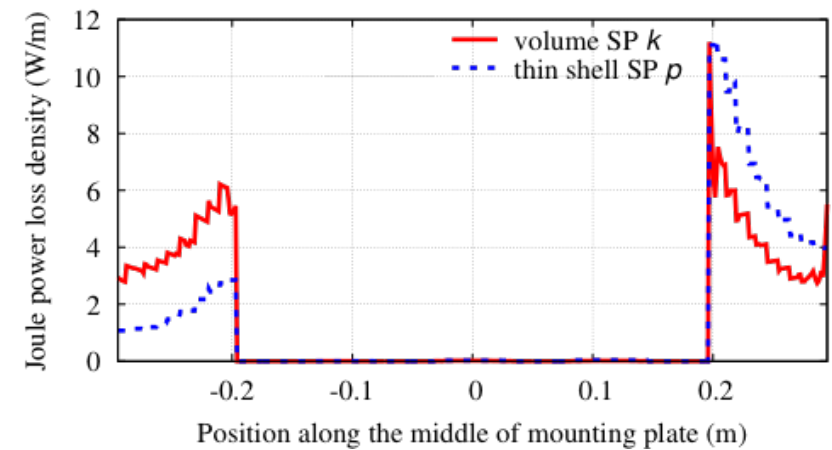

Figure 5. Joule power loss density for the TS and VS solution through the plate hole $\left(I_{\max }=2 k A\right)$.

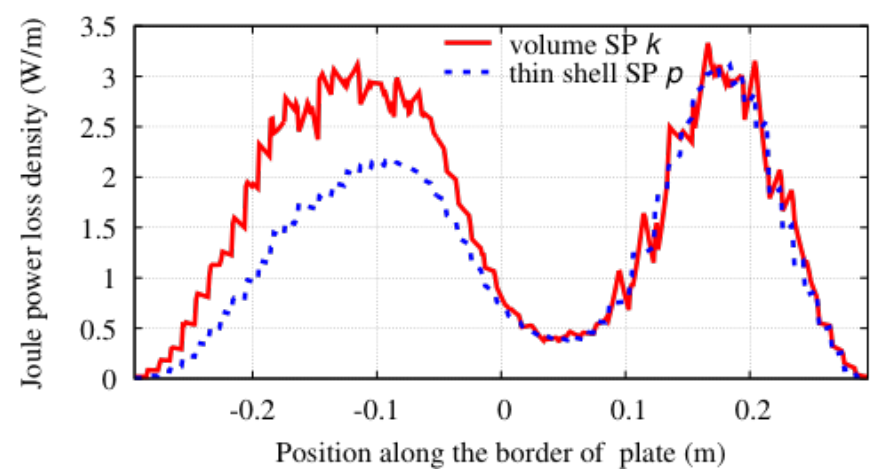

\begin{tabular}{|c|c|c|c|c|}
\hline \multirow{2}{*}{$\begin{array}{l}\text { Current } \\
I(\mathrm{kA})\end{array}$} & \multirow{2}{*}{$\begin{array}{c}\text { Frequency } \\
f(\mathrm{~Hz})\end{array}$} & \multicolumn{2}{|c|}{ Massive inductors } & \multirow{2}{*}{$\begin{array}{c}\text { Measured } \\
\text { values (W) }\end{array}$} \\
\hline & & $\begin{array}{l}\text { Thin shell } \\
\mathrm{P}_{\text {thin }}(\mathrm{W})\end{array}$ & $\begin{array}{l}\text { Volume } \\
\mathrm{P}_{\mathrm{vol}}(\mathrm{W})\end{array}$ & \\
\hline 2000 & 50 & 51.8 & 62.58 & 65 \\
\hline 2250 & 50 & 64.8 & 78.9 & 74 \\
\hline 2500 & 50 & 80.8 & 97.5 & 95 \\
\hline 2800 & 40 & 100.5 & 122.7 & 119 \\
\hline
\end{tabular}

\section{Conclusions}

\section{References}

[2] Vuong Q. Dang, P. Dular, R.V. Sabariego, L. Krähenbühl, C. Geuzaine, "Subproblem approach for Thin Shell Dual Finite Element Formulations," IEEE Trans. Magn., vol. 48, no. 2, pp. 407-410, 2012.

[3] P. Dular, Vuong Q. Dang, R. V. Sabariego, L. Krähenbühl and C. Geuzaine, "Correction of thin shell finite element magnetic models via a subproblem method," IEEE Trans. Magn., Vol. 47, no. 5, pp. 158 -1161, 2011.

[4] Dang Quoc Vuong "A Subproblem Method for Accurate Thin Shell Models between Conducting and Non-Conducting Regions," The University of Da Nang Journal of Science and Technology, no 12 (109).2016.

[5] Tran Thanh Tuyen, Dang Quoc Vuong, Bui Duc Hung and Nguyen The Vinh "Computation of magnetic fields in thin shield magetic models via the Finite Element Method," The University of Da Nang Journal of Science and Technology, no 7 (104).2016.

[6] Dang Quoc Vuong "Modeling of Electromagnetic Systems by Coupling of Subproblems - Application to Thin Shell Finite Element Magnetic Models," $\mathrm{PhD}$. Thesis (2013/06/21), University of Liege, Belgium, Faculty of Applied Sciences, June 2013.

[7] Dang Quoc Vuong, Bui Duc Hung and Khuong Van Hai "Using Dual Formulations for Correction of Thin Shell Magnetic Models by a Finite Element Subproblem Method," The University of Da Nang Journal of Science and Technology, no 6 (103).2016. 
[8] S.V. Kulkarni, J.C. Olivares, R. Escarela-Prerez, V. K. Lakhiani and J. Turowski "Evaluation of Eddy Current Losses in the Cover Plates of Distribution Transformers," IEE Proc. Sci. Meas. Technol, Vol. 151, No. 5, September 2004.

[9] Dang Quoc Vuong "An iterative subproblem method for thin shell finite element magnetic models," The University of Da Nang Journal of Science and Technology, no 12 (121).2017.

[10] Tran Thanh Tuyen and Dang Quoc Vuong, "Using a Magnetic Vector Potential Formulation for Calculting Eddy Currents in Iron Cores of Transformer by A Finite Element Method," The University of Da Nang Journal of Science and Technology, no 3 (112), 2017 (Part I).

[11] S. Koruglu, P. Sergeant, R.V. Sabarieqo, Vuong. Q. Dang, M. De Wulf "Influence of contact resistance on shielding efficiency of shielding gutters for high-voltage cables," IET Electric Power Applications, Vol.5, No.9, (2011), pp. 715-720 